Gynäkologische Endokrinologie 2013 .

11:248-255

DOI 10.1007/s10304-013-0562-6

Online publiziert: 11. September 2013

๑) Springer-Verlag Berlin Heidelberg 2013

Redaktion

M. Birkhäuser, Basel
K. Feller-Zulueta $\cdot$ E. Christ

Poliklinik für Endokrinologie, Diabetologie und Klinische Ernährung,

Inselspital, Universitätsspital Bern, Schweiz

\title{
Allgemein endokrinologische Probleme bei der Frau über 70 Jahre
}

Im Lauf des 20. Jahrhunderts ist in den westlichen Industrienationen der Anteil der älteren Menschen an der Gesamtbevölkerung stetig gestiegen [18]. Während es zahlreiche Studien über physiologische und pathologische Veränderungen rund um die Reproduktion gibt, sind Studien über andere endokrine Veränderungen im Alter rar. Komplizierend kommt hinzu, dass sich der physiologische Alterungsprozess auch zum Teil in veränderten Hormonspiegeln widerspiegeln kann, was die Abgrenzung zu behandlungsbedürftigen Endokrinopathien erschwert. In der folgenden Übersichtsarbeit haben wir versucht, wesentliche Abklärungsschritte und Therapiemodalitäten der Endokrinopathien der älteren Frau zusammenzutragen.

\section{Diabetes mellitus Typ 2 und metabolisches Syndrom \\ Diabetes mellitus Typ 2}

Diabetes mellitus Typ 2 ist im Alter häufig. Im Alterssegment der 65- bis 74-Jährigen ist schon heute einer von 7 Patienten zuckerkrank [10]. Neben einer genetischen Disposition sind der Mangel an Bewegung und das Übergewicht pathogenetisch entscheidend.

Der Stellenwert einer individualisierten Diabetestherapie, abgestimmt auf die Komorbiditäten der Patientin, nimmt im Alter zu.

Alle Behandlungskonzepte sollten Hypoglykämien nach Möglichkeit vermeiden.
Insulin ist das anabole Hormon schlechthin und trägt zum Erhalt der Muskelmasse bei, was insbesondere bei der gebrechlichen Patientin von Bedeutung ist.

Screening und Diagnosestellung eines Diabetes mellitus sind seit 2009 mit der Einführung des $\mathrm{HbA}_{\mathrm{lc}}$ als Kriterium einfacher geworden. Die aktuellen Diagnosekriterien für den manifesten Diabetes und den Prädiabetes sind in $\bullet$ Tab. 1 aufgelistet. Die Entwicklung vom normalen Insulinstoffwechsel zum manifesten Diabetes mellitus stellt ein Kontinuum dar. Deshalb sind bereits bei Diagnose eines Prädiabetes mellitus Lifestyle-Interventionen indiziert [20].

Bei Neudiagnose eines Diabetes mellitus sollte eine Patientin in der Blutzuckermessung geschult werden und eine Ernährungsberatung erhalten. Erstere beinhaltet das gezielte Blutzuckermessen in Form von Tagesprofilen (3-mal präprandial, 1-mal postprandial), und Letztere hat zum Ziel, bei Übergewicht die Zufuhr energiereicher Nahrung (z. B. Fette) $\mathrm{zu}$ vermindern und kohlenhydrathaltige Speisen und Getränke mit raschem Blutzuckeranstieg nach Einnahme (z. B. Süßgetränke) quantitativ zu reduzieren [20]. An Impfungen sind die jährliche Grippen- und die Pneumokokkenimpfung empfohlen.

Die Therapieziele bei betagten Diabetikerinnen sollten individuell festgelegt werden. Bei rüstigen Patientinnen ohne diabetische Spätkomplikationen und mit einer Lebenserwartung $>10$ Jahre wird wie bei jüngeren Patientinnen ein $\mathrm{HbA}_{1 \mathrm{c}}$-Ziel von $\leq 7 \%$ angestrebt. Bei gebrechlichen Patientinnen mit multiplen Komorbiditäten und einer Lebenserwartung von weniger als 10 Jahren kann der
Ziel-HbA $\mathrm{Ac}_{1 \mathrm{c}}$-Wert auf 7,5-8\% angehoben werden. Alle Behandlungskonzepte sollten Hypoglykämien vermeiden, da diese insbesondere bei älteren Patientinnen mit erhöhtem Sturzrisiko sowie dem Auftreten eines akuten Koronarsyndroms, Herzrhythmusstörungen und zerebrovaskulärem Insult assoziiert sein können. Umgekehrt sollten aber $\mathrm{HbA}_{1 \mathrm{c}}$-Werte deutlich über $8 \%$ auch bei polymorbiden Patientinnen vermieden werden, da sie meist mit einer katabolen Stoffwechsellage einhergehen und somit zu Muskel- und Fettabbau führen [20].

Ein jährliches Screening für diabetische Spätkomplikationen ist auch bei betagten Patientinnen wichtig. Dazu gehören die ophthalmologische Untersuchung (Retinopathie, Glaukom, Katarakt), die Bestimmung der Mikroalbuminurie sowie regelmäßige Fußinspektionen. Letztere sind besonders wichtig angesichts der zunehmenden Prävalenz der peripheren Polyneuropathie im Alter sowie der Tatsache, dass über $30 \%$ der älteren Diabetespatienten ihre Füße nicht richtig untersuchen können [19].

Die Therapie kardiovaskulärer Korisikofaktoren umfasst die Motivation zum Rauchstopp, die Kontrolle einer arteriellen Hypertonie (Ziel-Blutdruck 130140/80-90) und die Therapie einer Dyslipidämie. Die Korrelation zwischen kardiovaskulärer Mortalität und totalem Cholesterin nimmt mit zunehmendem Alter ab. Ein tiefes Gesamtcholesterin kann sogar als Zeichen der Malnutrition ein Prädiktor für eine erhöhte Mortalität sein [10]. Die Empfehlungen zur Statintherapie der Arbeitsgruppe Fortbildung Endokrinologie-Diabetologie sind in $\bullet$ Tab. 2 zusammengefasst. Eine Thrombozytenaggre- 


\section{Infobox 1 Diagnosekriterien des metabolischen Syndroms gemäß ATPIII-Richtlinien}

Drei der 5 Kriterien müssen erfüllt sein:

1. Abdominale Adipositas (bei Frauen Bauchumfang $>88 \mathrm{~cm}$ )

2. Serumtriglyzeride $>1,7 \mathrm{mmol} / \mathrm{l}$ oder medikamentöse Therapie einer Hypertriglyzeridämie

3. HDL-Cholesterin bei Frauen $<1,3 \mathrm{mmol} / \mathrm{l}$ oder Statintherapie

4. Blutdruck $\geq 130 / 85 \mathrm{mmHg}$

5. Nüchtern-Blutzucker $>5,6 \mathrm{mmol} / \mathrm{l}$ oder antidiabetische Therapie

gationshemmung mit Acetylsalicylsäure wird zur Sekundärprävention kardiovaskulärer Krankheiten empfohlen.

Der von den amerikanischen und europäischen Fachgesellschaften empfohlene Algorithmus für die medikamentöse antidiabetische Therapie wird in $\bullet$ Tab. 3 dargestellt. Die $\bullet$ Abb. 1 zeigt die Wirkmechanismen der Substanzklassen. Bei der Wahl eines geeigneten oralen Antidiabetikums müssen sowohl mögliche Komorbiditäten wie Nieren-, Herz- und Leberinsuffizienz sowie Osteoporose als auch Medikamenteninteraktionen oder geplante Kontrastmitteluntersuchungen berücksichtigt werden [10]. Es ist zu bedenken, dass der Nachweis der Mortalitätssenkung bisher einzig für Lifestyle, Metformin und Insuline erbracht worden ist, jedoch nicht für die neuen Antidiabetika.

\section{Metabolisches Syndrom}

Der Begriff „metabolisches Syndrom“ bezeichnet das gleichzeitige Auftreten von Risikofaktoren sowohl für die Entwicklung eines Diabetes mellitus Typ 2 als auch kardiovaskulärer Erkrankungen. Die am häufigsten verwendete Definition ist diejenige der National Cholesterol Education Program/Adult Treatment Panel (NCEP/ATP III), die in $\bullet$ Infobox 1 dargestellt ist.

Das metabolische Syndrom ist im Alter häufig und betrifft rund $44 \%$ der über 70-jährigen Frauen [5]. Die Definition dieses Syndroms und die Bedeutung in der Diagnose werden kontrovers diskutiert. Sicher ist, dass beim Vorliegen eines metabolischen Syndroms die frühzeitige Intensivierung von Lifestyle-Interventionen gerechtfertigt ist.

\section{Schilddrüse}

Die Prävalenz von Schilddrüsenerkrankungen steigt mit dem Alter. Hyper- oder hypothyreote Stoffwechsellagen können bei älteren Patientinnen mit atypischen Symptomen einhergehen. Eine Hyperthyreose sollte insbesondere aufgrund der möglichen kardialen und ossären Nebenwirkungen behandelt werden, die Therapiemodalitäten sind individuell festzulegen. Die Hypothyreose ist mit einer Hypercholesterolämie assoziiert, die zum erhöhten kardiovaskulären Risiko beiträgt.

Das Schilddrüsenvolumen erhöht sich leicht mit dem Alter. Schilddrüsenhormonproduktion und Clearance verringern sich gleichzeitig, sodass sich die freien Schilddrüsenhormonkonzentrationen mit zunehmendem Alter nicht ändern (• Tab. 4). Dies gilt auch für das $\mathrm{fT}_{3}$. Sobald allerdings Patientinnen mit assoziierten Krankheiten ausgewählt werden - eine häufige Konstellation bei Patientinnen $>70$ Jahre - können tiefe $\mathrm{fT}_{3^{-}}$ Spiegel gemessen werden. Diesem Phänomen liegt eine Reduktion der peripheren Umwandlung von $\mathrm{fT}_{4} \mathrm{zu} \mathrm{fT}_{3}$ zugrunde, die durch die Komorbidität (Infekt, konsumierende Krankheit) bedingt ist (sog. "nonthyroidal illness"). Die Schilddrüsenwerte sollten deshalb bei akuten Krankheiten zurückhaltend gemessen werden. Eine Schilddrüsenhormontherapie aufgrund von Schilddrüsenwerten, die anlässlich einer akuten Krankheit gemessen werden, ist nicht zu empfehlen. Die - Abb. 2 gibt einen Überblick über den Schilddrüsenregelkreis.

\section{Überfunktion - Hyperthyreose und subklinische Hyperthyreose}

Die manifeste und subklinische Hyperthyreose ist ein klinisches Zustandsbild. Dahinter verbirgt sich diagnostisch meist ein Morbus Basedow (klinisch suggestiv bei anderen Autoimmunerkrankungen und/oder nachgewiesener Orbitopathie) oder eine Autonomie (auch unter dem Begriff „autonomes Adenom“ respektive „toxisches Adenom“ bekannt). Ein Bei- spiel ist in - Abb. 3 dargestellt. Im Alter ist der Morbus Basedow selten, während die Prävalenz autonomer Knoten mit/ohne Struma zunimmt. Jod-induzierte Hyperthyreosen infolge Kontrastmittelexposition bei bis dahin unbekannter Autonomie oder Amiodaron-Therapie sind im Alter deshalb häufiger.

Die Symptome der Hyperthyreose manifestieren sich bei älteren Patientinnen im kardiovaskulären (Vorhofflimmern, Herzinsuffizienz, koronare Herzerkrankung) oder zentralnervösen System (Apathie, Verwirrung, Abgeschlagenheit). Typische hypermetabole Symptome (Wärmeintoleranz, Schwitzen) können fehlen. Gastrointestinale Symptome sind unspezifisch. Der hyperkatabole Zustand kann zu einem Muskelschwund führen, was eine Erhöhung des Sturzrisikos zur Folge hat. Eine Hyperthyreose ist mit einer Abnahme der Knochendichte und mit Frakturen assoziiert und sollte bei entsprechendem Verdacht gezielt gesucht werden.

\section{$\nabla$}

Bei Vorhofflimmern und Osteoporose sollen bei älteren Patientinnen Schilddrüsenparameter gemessen werden.

Dabei unterschiedet man die manifeste Hyperthyreose (supprimiertes TSH und erhöhte $\mathrm{fT}_{4}$ - und/oder $\mathrm{fT}_{3}$-Konzentrationen) von der subklinischen Hyperthyreose (supprimiertes oder zu tiefes TSH bei normalen $\mathrm{fT}_{4}$ - und $\mathrm{fT}_{3}$-Konzentrationen). Die zugrunde liegenden Diagnosen sind prinzipiell dieselben: Morbus Basedow und Autonomie. Während die Diagnose des Morbus Basedow klinisch und mittels positiven TSH-Rezeptor-Antikörpers (häufig auch positive TPO-AK) gestellt werden kann, sind die Antikörper bei der Autonomie negativ, und die Szintigraphie ( $\bullet$ Abb. 3 ) ist in dieser Situation hilfreich. Bei einer Amiodaron-induzierten Hyperthyreose ist typischerweise $\mathrm{fT}_{4}$ deutlich stärker erhöht als $\mathrm{fT}_{3}$, da die periphere Konversion von $\mathrm{T}_{4} \mathrm{zu}_{3}$ durch Amiodaron inhibiert wird. Die Therapiemodalitäten sind für subklinische und manifeste Hyperthyreose identisch. Prinzipiell kann thyreostatisch behandelt werden, in erster Linie mit Carbimazol, das eine längere Wirkdauer (erlaubt eine 1-mal tägliche Dosierung), einen ra- 
scheren Wirkungseintritt sowie eine tiefere Inzidenz an Nebenwirkungen als Propylthiouracil hat. Thyreostatika können bei älteren Patientinnen zu Nebenwirkungen (Hautmanifestationen, Hepatitis und selten Leukopenie) führen und gehen bei Autoimmunthyreopathien mit einem ca. 50\% hohen Rezidivrisiko nach einer 1-jährigen Therapie einher [3]. Bei einer Autonomie ist eine lebenslängliche Therapie erforderlich. Die Behandlung der sympathikotonen Hyperthyreosezeichen erfolgt meist mit einem $\beta$-Blocker. Günstig ist Propranolol, das zusätzlich die Konversion von $\mathrm{T}_{4} \mathrm{zu} \mathrm{T}_{3}$ hemmt. Die totale Thyreoidektomie tritt bei älteren und polymorbiden Patientinnen aufgrund des Narkose- und Operationsrisikos etwas in den Hintergrund. Die Radiojodablation ist einfach in der Durchführung und nebenwirkungsarm und somit gerade bei älteren und polymorbiden $\mathrm{Pa}$ tientinnen die Therapie der Wahl. Bei allen definitiven Therapieoptionen (Chirurgie, Radiojodablation) ist im Anschluss eine meist lebenslängliche LevothyroxinSubstitution erforderlich.

\section{Unterfunktion - Hypothyreose und subklinische Hypothyreose}

Die manifeste und subklinische Hypothyreose ist ein klinisches Zustandsbild. Die zugrunde liegende häufigste Diagnose ist die Hashimoto-Thyreoiditis [17], gefolgt von einem Zustand nach Thyreoidektomie oder Radiojodablation sowie medikamentös induzierte Hypothyreosen (z. B. Amiodarion, Lithium, Interferon- $\alpha$ ).

Hypothyreosen werden gerade bei älteren Patientinnen leicht übersehen, da sie mit unspezifischen Beschwerden einhergehen, die im Alter auch bei euthyreoten Individuen vorkommen. Kardiovaskuläre (z. B. Herzinsuffizienz, Angina pectoris) und neurologische Symptome wie kognitive Beeinträchtigungen können bei der alten Patientin Ausdruck einer Hypothyreose sein. Im Status können verzögerte muskuläre Eigenreflexe, Myxödem, Heiserkeit oder trockene Hautverhältnisse auffallen.

Bei einer manifesten Hypothyreose zeigen sich laboranalytisch ein erhöhtes TSH und ein zu tiefes $\mathrm{fT}_{4}$. Bei einer subklinischen Hypothyreose ist das TSH erhöht bei normalem $\mathrm{fT}_{4}$. Insbesondere letztere Konstellation ist bei postmenopausalen Frauen häufig (bis zu 8\%; [13]). Bei einer Hashimoto-Thyreoiditis finden sich zusätzlich Antikörper gegen die thyreoidale Peroxidase (Anti-TPO-Antikörper) in bis zu 90\% der Fälle. Hypothyreosen können mit Dyslipidämien assoziiert sein (typischerweise Erhöhung von Gesamt- und LDL-Cholesterin) und gehen mit einer Erhöhung des kardiovaskulären Risikos einher.

Eine manifeste Hypothyreose und eine subklinische Hypothyreose mit TSH $>10$ mU/1 [15] werden mit einer Levothyroxin-Substitutionstherapie behandelt. Bei einer subklinischen Hypothyreose mit TSH-Konzentration zwischen 4,5 und $10 \mathrm{mU} / \mathrm{l}$ ist die Therapieindikation noch unklar und muss - nach einer erneuten Messung - individuell festgelegt werden. Bei älteren Patientinnen und beim Vorliegen kardiovaskulärer Komorbiditäten empfiehlt sich eine Startdosis von $50 \mu \mathrm{g} /$ Tag Levothyroxin, ansonsten kann mit einer Startdosis von 75-100 $\mu \mathrm{g} / \mathrm{Tag}$ begonnen werden. Eine Überprüfung von $\mathrm{TSH}$ und $\mathrm{fT}_{4}$ sollte aufgrund der langen Halbwertszeit frühestens nach 4 bis 6 Wochen erfolgen. Die auf dem Markt befindlichen Präparate unterscheiden sich nur in der verfügbaren Dosis.

\section{Schilddrüsenknoten und Schilddrüsenkarzinom}

Die Prävalenz von Schilddrüsenknoten beträgt bei älteren Frauen bis zu 40\% [5]. Diese können im Rahmen einer Struma (= Schilddrüsenvergrößerung), multipel oder singulär auftreten. Nur bei erniedrigtem TSH soll eine Szintigraphie durchgeführt werden. Bei vermehrter Jodaufnahme („warmer Knoten“) ist die entsprechende Läsion sicher nicht maligne. Auf der anderen Seite ist eine verminderte Jodaufnahme („,kalter Knoten“) noch lange nicht maligne, da z. B. Zysten im Szintigramm ebenfalls als „kalt“ imponieren. Deshalb drängt sich bei Patientinnen mit Schilddrüsenknoten, die $>1-1,5 \mathrm{~cm}$ im Durchmesser messen, eine weitere Abklärung mittels Feinnadelpunktion auf. Dabei muss allerdings der Gesamtkontext der Patientin (Komorbiditäten) bei
Gynäkologische

Endokrinologie 2013 · 11:248-255

DOI 10.1007/s10304-013-0562-6

(c) Springer-Verlag Berlin Heidelberg 2013

\section{K. Feller-Zulueta $\cdot$ E. Christ Allgemein endokrinologische Probleme bei der Frau über 70 Jahre}

\section{Zusammenfassung}

Die häufigsten endokrinen Erkrankungen bei der Frau über 70 Jahre sind das metabolische Syndrom mit oder ohne Typ-2-Diabetes, Schilddrüsenfunktionsstörungen sowie Schilddrüsenknoten. Alle übrigen endokrinen Pathologien sind selten, führen aber wegen langer Latenz der Diagnose häufig zu einer Einschränkung der Lebensqualität. Der vorliegende Beitrag erläutert wesentliche Abklärungsschritte und Therapiemodalitäten der Endokrinopathien der älteren Frau.

\section{Schlüsselwörter}

Endokrinopathien - Diabetes mellitus

Typ 2 - Schilddrüsenfunktionsstörungen . Schilddrüsenknoten · Ältere Frauen

\section{General endocrinological problems in women aged over 70 years}

\section{Abstract}

The most common endocrine disorders in women over 70 years old are the metabolic syndrome with or without type 2 diabetes mellitus, thyroid dysfunction and thyroid nodules. All other endocrine pathologies are rare but can lead to impaired quality of life due to delay in diagnosis. This report provides information on the essential diagnostic steps and therapy modalities of endocrine disorders in elderly females.

\section{Keywords}

Endocrine disorders - Diabetes mellitus type $2 \cdot$ Thyroid dysfunction - Thyroid nodule . Elderly women

der weiteren Abklärung in die Entscheidung mit einbezogen werden.

$>$ 90\% der Knoten benigne. Unter differenzierten Schilddrüsenkarzinomen werden das papilläre (häufigstes Schilddrüsenkarzinom; betrifft eher jüngere Patientinnen) und das follikuläre Schilddrüsenkarzinom (15\% der Schilddrüsenkarzinome; gehäuft bei älteren $\mathrm{Pa}$ tientinnen) verstanden. Beide haben eine 
Tab. 1 Diagnosekriterien des Diabetes mellitus

\begin{tabular}{llll}
\hline Stadium & $\mathbf{H b A}_{\mathbf{1 c}}(\mathbf{\%})$ & $\begin{array}{l}\text { Nüchtern-Glukose } \\
(\mathbf{m m o l} / \mathbf{l})\end{array}$ & $\begin{array}{l}\text { 2-h-Glukose im OGTT } \\
(\mathbf{m m o l} / \mathbf{l})\end{array}$ \\
\hline Diabetes mellitus & $\geq 6,5$ & $\geq 7,0$ & $\geq 11,1$ \\
\hline Prädiabetes mellitus & $5,7-6,4$ & $5,6-6,9$ & $7,8-11,0$ \\
\hline $\begin{array}{l}\text { Ein Glukosewert }>11,1 \text { mmol// mit entsprechender Klinik sichert die Diagnose. Bei grenzwertigem Wert sollte } \\
\text { der Wert durch eine Wiederholungsbestimmung gesichert werden. } \\
\text { OGTT oraler Glukosetoleranztest. }\end{array}$ & & \\
\hline
\end{tabular}

Tab. 2 Empfehlungen zum Einsatz von Statinen bei der älteren Diabetikerin. (Aus: [10])

\begin{tabular}{|c|c|c|}
\hline Primärprävention & Sekundärprävention & Keine Prävention \\
\hline $\begin{array}{l}\text { - LDL-Cholesterin }>3,5 \mathrm{mmol} / \mathrm{l} \\
\text { und }\end{array}$ & $\begin{array}{l}\text { - LDL-Cholesterin }>2,6 \mathrm{mmol} / \mathrm{l} \\
\text { und }\end{array}$ & $\begin{array}{l}\text { - Schlechter Allgemeinzustand } \\
\text { - Kontraindikation für Statine }\end{array}$ \\
\hline $\begin{array}{l}\text { - } 1 \text { weiterer kardiovaskulärer } \\
\text { Risikofaktor }\end{array}$ & - Biologisches Alter <80 Jahre & $\begin{array}{l}\text { - Pflegebedürftigkeit } \\
\text { - Demenz }\end{array}$ \\
\hline - Biologisches Alter $<80$ Jahre & & \\
\hline
\end{tabular}

Tab. 3 Antidiabetika bei älteren Patientinnen mit Diabetes. (Adaptiert nach [10])

\begin{tabular}{|c|c|c|c|c|}
\hline $\begin{array}{l}\text { Stoffklasse } \\
\text { Name }\end{array}$ & $\begin{array}{l}\text { Hypoglykämie- } \\
\text { risiko }\end{array}$ & $\begin{array}{l}\text { Nebenwirkun- } \\
\text { gen }\end{array}$ & $\begin{array}{l}\text { Niereninsuffi- } \\
\text { zienz }\end{array}$ & Bemerkungen \\
\hline $\begin{array}{l}\text { Biguanide } \\
\text { Metformin } \\
\text { (Metfin }^{\circledast}, \\
\left.\text { Glucophage }^{\circledast}\right)\end{array}$ & - & $\begin{array}{l}\text { Gastrointestinale } \\
\text { Beschwerden } \\
\text { (Blähungen, } \\
\text { Diarrhö) }\end{array}$ & $\begin{array}{l}\text { Ab Clearance } \\
<60 \mathrm{ml} / \mathrm{min} \text { nicht } \\
\text { empfohlen; } \\
\text { Gefahr der } \\
\text { Laktatazidose }\end{array}$ & $\begin{array}{l}\text { Keine Hypoglyk- } \\
\text { ämien } \\
\text { Gefahr Gewebe- } \\
\text { hypoxie, z. B. } \\
\text { Herzinsuffizienz; } \\
\text { Stopp vor Rönt- } \\
\text { genkontrastmit- } \\
\text { teluntersuchung }\end{array}$ \\
\hline $\begin{array}{l}\text { Sulfonylharn- } \\
\text { stoffe } \\
\text { Glimepirid } \\
\left(\text { Amary }{ }^{\circledR}\right) \\
\text { Gliclazid } \\
\left(\text { Diamicron } M R^{\oplus}\right)\end{array}$ & ++ & $\begin{array}{l}\text { Gewichtszunah- } \\
\text { me } \\
\text { Gastrointestinale } \\
\text { Nebenwirkungen } \\
\text { Hautreaktionen }\end{array}$ & $\begin{array}{l}\text { Verlängerte } \\
\text { Wirkung, erhöhte } \\
\text { Hypoglykämie- } \\
\text { gefahr }\end{array}$ & $\begin{array}{l}\text { Bekanntes } \\
\text { Effizienz- und Si- } \\
\text { cherheitsprofil } \\
\text { Cave: Medika- } \\
\text { menteninterak- } \\
\text { tionen }\end{array}$ \\
\hline $\begin{array}{l}\text { Glinide } \\
\text { Repaglinid } \\
\left(\text { Novonorm }^{\oplus}\right) \\
\text { Nateglinid } \\
\left(\text { Starlix }^{\circledast}\right)\end{array}$ & + & $\begin{array}{l}\text { Erhöhte Leber- } \\
\text { werte } \\
\text { Hautreaktionen }\end{array}$ & $\begin{array}{l}\text { Prinzipiell bei } \\
\text { Niereninsuffi- } \\
\text { zienz möglich } \\
\text { (Beginn mit klei- } \\
\text { ner Dosis) }\end{array}$ & $\begin{array}{l}\text { Kein Glinid bei } \\
\text { Nahrungskarenz } \\
\text { Weniger potent } \\
\text { als Sulfonylharn- } \\
\text { stoffe }\end{array}$ \\
\hline $\begin{array}{l}\text { DPP-IV-Inhibi- } \\
\text { toren } \\
\text { Sitagliptin } \\
\left.\text { (Januvia }^{\oplus}\right) \\
\text { Vildagliptin } \\
\text { (Galvus }^{\circledR} \text { ) }\end{array}$ & - & $\begin{array}{l}\text { Gastrointestinale } \\
\text { Nebenwirkungen }\end{array}$ & $\begin{array}{l}\text { Dosisreduktion } \\
\text { bei Januvia }{ }^{\circledast} \text { und } \\
\text { Galvus }{ }^{\circledast} \text { bei } \\
\text { schwerer Nieren- } \\
\text { insuffizienz }\end{array}$ & $\begin{array}{l}\text { Limitierte Lang- } \\
\text { zeitdaten bezüg- } \\
\text { lich Effizienz und } \\
\text { Sicherheit }\end{array}$ \\
\hline $\begin{array}{l}\text { GLP-1-Agonisten } \\
\text { Exenatide } \\
\left(\text { Byetta }^{\oplus}\right) \\
\text { Liraglutid } \\
\left(\text { Victoza }^{\oplus)}\right.\end{array}$ & - & $\begin{array}{l}\text { Gastrointestinale } \\
\text { Nebenwirkungen } \\
\text { Pankreatitis }\end{array}$ & $\begin{array}{l}\text { Nur bei termina- } \\
\text { ler Niereninsuf- } \\
\text { fizienz erhöhte } \\
\text { Halbwertszeit }\end{array}$ & $\begin{array}{l}\text { Limitierte Lang- } \\
\text { zeitdaten bezüg- } \\
\text { lich Effizienz und } \\
\text { Sicherheit } \\
\text { Gewichtsabnah- } \\
\text { me } \\
\text { s.c.-Injektion }\end{array}$ \\
\hline $\begin{array}{l}\text { Insulin } \\
\text { Diverse humane } \\
\text { Insuline und Ana- } \\
\text { loga }\end{array}$ & + & & $\begin{array}{l}\text { Therapie der } \\
\text { Wahl bei Nieren- } \\
\text { insuffizienz }\end{array}$ & $\begin{array}{l}\text { Blutzuckermes- } \\
\text { sung, Injektion } \\
\text { Bekanntes } \\
\text { Effizienz- und } \\
\text { Sicherheitsprofil }\end{array}$ \\
\hline
\end{tabular}

Aufgrund ihrer ossären, urogenitalen und kardialen Nebenwirkungen haben die Glitazone in der Behandlung älterer Diabetikerinnen aus unserer Sicht nur noch in Ausnahmefällen einen Platz. sehr gute Prognose, wobei das follikuläre Karzinom bezüglich Mortalität prognostisch etwas schlechter ist als das papilläre. Differenzierte Schilddrüsenkarzinome können primär chirurgisch und bei Bedarf mittels Radiojodablation gezielt angegangen werden. Das anaplastische Schilddrüsenkarzinom tritt bei älteren Patienten auf, ist lokal invasiv, metastasiert rasch und ist mit einer sehr schlechten Prognose vergesellschaftet.

\section{Nebennierenrinde}

\section{Cortisol und Aldosteron}

Die durchschnittliche 24-h-Cortisol-Konzentration im Serum nimmt im Alter um $20-50 \% \mathrm{zu}$ [16]. Es handelt sich um interindividuell unterschiedliche Veränderungen, denen im Rahmen akuter Erkrankungen wahrscheinlich keine Bedeutung zukommt, die jedoch möglicherweise chronische Auswirkungen haben könnten. So wird die Häufung an Schlafstörungen bei betagten Personen mit erhöhtem abendlichem Cortisolspiegel in Verbindung gebracht. Ebenso findet sich eine Assoziation zwischen höherer Cortisolsekretion und abnehmender Gedächtnisleistung sowie erhöhter Inzidenz von Frakturen [4, 14]. Der Nüchtern-Cortisolwert morgens um 08:00 gemessen spiegelt am Zuverlässigsten die Nebennierenfunktion wider und ist altersunabhängig (• Tab. 4).

Die Sekretion sowie die Serumkonzentration von Aldosteron sinken im Alter als Folge einer reduzierten Reninsekretion [2]. Dies kann insbesondere bei Patientinnen mit einem Diabetes mellitus und verminderter Trinkmenge bei eingeschränkter Nierenfunktion in einen hyporeninämischen Hypoaldosteronismus mit gefährlicher Hyperkaliämie und metabolischer Azidose münden. Besonders gefährdet sind Patientinnen mit gleichzeitiger Blockade des Renin-Angiotensin-Aldosteron-Systems (Antihypertensiva).

\section{Inzidentalome}

Am häufigsten sind im Alter die sog. Inzidentalome der Nebennieren. Darunter versteht man Raumforderungen der Nebenniere, die als Nebenbefund einer abdominalen Bildgebung gefunden wer- 
Tab. 4 Normwerte wichtiger Hormonkonzentrationen ${ }^{\text {a }}$ bei Patientinnen $>70$ Jahre

\begin{tabular}{|c|c|c|}
\hline Hormon & Normwerte & Bemerkungen \\
\hline \multicolumn{3}{|l|}{ Schilddrüse } \\
\hline $\mathrm{TSH}(\mathrm{mU} / \mathrm{l})$ & $0,35-4,50$ & Altersunabhängig \\
\hline $\mathrm{fT}_{4}(\mathrm{pmol} / \mathrm{l})$ & $9,5-25,0$ & Altersunabhängig \\
\hline $\mathrm{fT}_{3}(\mathrm{pmol} / \mathrm{l})$ & $2,9-6,5$ & Altersunabhängig \\
\hline \multicolumn{3}{|l|}{ Nebennieren } \\
\hline Cortisol (nmol/l) & $170-540$ & $\begin{array}{l}\text { Morgens nüchtern am zuver- } \\
\text { lässigsten, altersunabhängig }\end{array}$ \\
\hline DHEA-S $(\mu \mathrm{mol} / \mathrm{l})$ & $2,68-9,23$ & Altersabhängig \\
\hline Aldosteron (pmol/l) & $28-440$ & $\begin{array}{l}\text { Altersunabhängig, liegend } \\
\text { gemessen }\end{array}$ \\
\hline \multicolumn{3}{|l|}{ Hypophyse $^{b}$} \\
\hline IGF-1 (ng/ml) & $35-168$ & $\begin{array}{l}\text { Altersabhängig, Marker der } \\
\text { Wachstumshormonwirkung }\end{array}$ \\
\hline Prolaktin $(\mu \mathrm{g} / \mathrm{l})$ & $4,8-24,0$ & $\begin{array}{l}\text { Altersunabhängig; cave: Stress } \\
\text { und Medikamente }\end{array}$ \\
\hline Cortisol (nmol/l) & $170-540$ & $\begin{array}{l}\text { Morgens nüchtern am zuver- } \\
\text { lässigsten, altersunabhängig }\end{array}$ \\
\hline $\mathrm{fT}_{4}(\mathrm{pmol} / \mathrm{l})$ & $9,5-25,0$ & Altersunabhängig \\
\hline $\mathrm{fT}_{3}(\mathrm{pmol} / \mathrm{l})$ & $2,9-6,5$ & Altersunabhängig \\
\hline \multicolumn{3}{|c|}{$\begin{array}{l}\mathrm{fT}_{4} \text { und } \mathrm{fT}_{3} \text { freies } \mathrm{T}_{4} \text { respektive } \mathrm{T}_{3} \text {, IGF-1 }{ }_{\text {,insulin-like growth factor-1", DHEA-S Dehydroepiandrosteron-Sulfat. }} \\
\text { "Angegeben werden hier die Normwerte des chemischen Labors des Universitätsspitals Bern. Diese Normwerte } \\
\text { können je nach angewandter Methode leicht variieren. Ebenso können die Einheiten je nach Labor unter- } \\
\text { schiedlich sein. Die Hormone der gonadalen Achse sind nicht aufgeführt. 'Das hypophysäre ACTH wird nur } \\
\text { in speziellen Fällen gemessen und hat eine schwierige Präanalytik (Kühlung auf Eis). Das TSH ist bei bekannter } \\
\text { Pathologie der Hypophyse nicht interpretierbar. }\end{array}$} \\
\hline
\end{tabular}

den. Meist handelt es sich dabei um gutartige Veränderungen, die nur ab einer bestimmten Größe ( $>4 \mathrm{~cm}$ ), hormoneller Autonomie oder dokumentiertem Wachstum chirurgisch angegangen werden müssen.

\section{Überfunktion}

Der Hypercortisolismus ist im Alter selten, kann aber übersehen werden, da Symptome des metabolischen Syndroms (Hypertonie, Glukoseintoleranz, Gewichtszunahme) und Osteoporose bei betagten Patientinnen unspezifisch und im Rahmen des „normalen“ Alterungsprozesses häufig sind. Klinisch fallen allerdings beim Hypercortisolismus - im Unterschied zum metabolischen Syndrom - die proximale Myopathie (Mühe beim Treppensteigen, Atrophie) und die pergamentartige Haut mit häufig Hämatomen auf [11]. Da der endogene Hypercortisolismus (ACTH-abhängig und unabhängig) mit einer deutlich erhöhten Mortalität vergesellschaftet ist, ist eine rasche Abklärung in einem erfahrenen Zentrum wichtig.
Der primäre Hyperaldosteronismus aufgrund eines Aldosteron produzierenden Adenoms der Nebennierenrinde ist die wichtigste Form einer sekundären Hypertonie, labormäßig nicht zwingend, aber häufig mit Hypokaliämie und Hypernatriämie vergesellschaftet. Therapeutisch kann eine laparoskopische Adrenalektomie durchgeführt werden, alternativ ist insbesondere bei Polymorbidität im Alter die medikamentöse Therapie mit Aldosteronantagonisten möglich.

\section{Unterfunktion}

Typische Symptome einer Nebennierenrinden (NNR-)Insuffizienz wie Adynamie, Schwäche, Gewichtsverlust, Verwirrung und Arthralgien sind im Alter ebenfalls unspezifisch und auch ohne zugrunde liegenden Hypocortisolismus häufig [5]. Das spezifischste Zeichen einer primären NNR-Insuffizienz ist die Hyperpigmentation z. B. der Handlinien. Ursachen einer primären Nebenniereninsuffizienz sind die Autoimmunadrenalitis (Morbus Addison), bilaterale Karzinommetastasen (z. B. Bronchuskarzinom) oder Blutungen (unter oraler Antikoagulation).

\section{》) Das spezifischste Zeichen einer primären Neben- nierenrindeninsuffizienz ist die Hyperpigmentation}

Bei der sekundären Formen einer NNRInsuffizienz (ACTH-Insuffizienz) fehlt die Hyperpigmentation, die klinische Symptome sind im Allgemeinen diskreter, da die Renin-Aldosteron-Achse noch funktioniert. Die häufigste Ursache ist die Langzeitbehandlung mit Kortikosteroiden, seltenere Ursache ist die Insuffizienz des Hypophysenvorderlappens (infolge eines Hypophysenmakroadenoms).

Typische Laborbefunde können neben dem erniedrigten Nüchtern-Cortisol und dem verminderten Cortisolanstieg nach ACTH-Gabe (Synacthen-Test) eine Hyponatriämie oder Hyperkaliämie, eine Lymphozytose und Eosinophilie sein.

Die Substitutionstherapie der primären Nebenniereninsuffizienz erfolgt mit Hydrokortison (Tagesdosis 15-25 mg Hydrokortison in 2 bis 3 Dosen, selten Prednison 5-7,5 mg 1-mal/Tag) in Kombination mit Fludrocortison (z. B. 1-mal $0,1 \mathrm{mg} / \mathrm{Tag})$, bei hypophysärer Insuffizienz wird lediglich Hydrocortison verschrieben. Bester Verlaufsparameter ist die Klinik. Alle Patientinnen brauchen einen Notfallausweis, damit in außergewöhnlichen Situationen (Unfall, hohes Fieber) die Cortisolsubstitution angepasst werden kann.

\section{Dehydroepiandrosteron}

Dehydroepiandrosteron (DHEA) und Dehydroepiandrosteron-Sulfat (DHEA-S) sind Vorläufer von Androgenen. Bis jetzt ist kein spezifischer Rezeptor für dieses Hormon bekannt, sodass man davon ausgeht, dass die Wirkung des DHEAs durch die periphere Konversion in Androgene erfolgt [9]. Die DHEA-Sekretion nimmt bereits ab der 30. Dekade kontinuierlich ab, die Serumkonzentration von DHEA beträgt bei Patientinnen über 70 Jahre noch ca. $20 \%$ derjenigen von 20 bis 30-Jährigen (• Tab. 4, [9]). Bis jetzt konnte aber in keiner der prospektiven, 


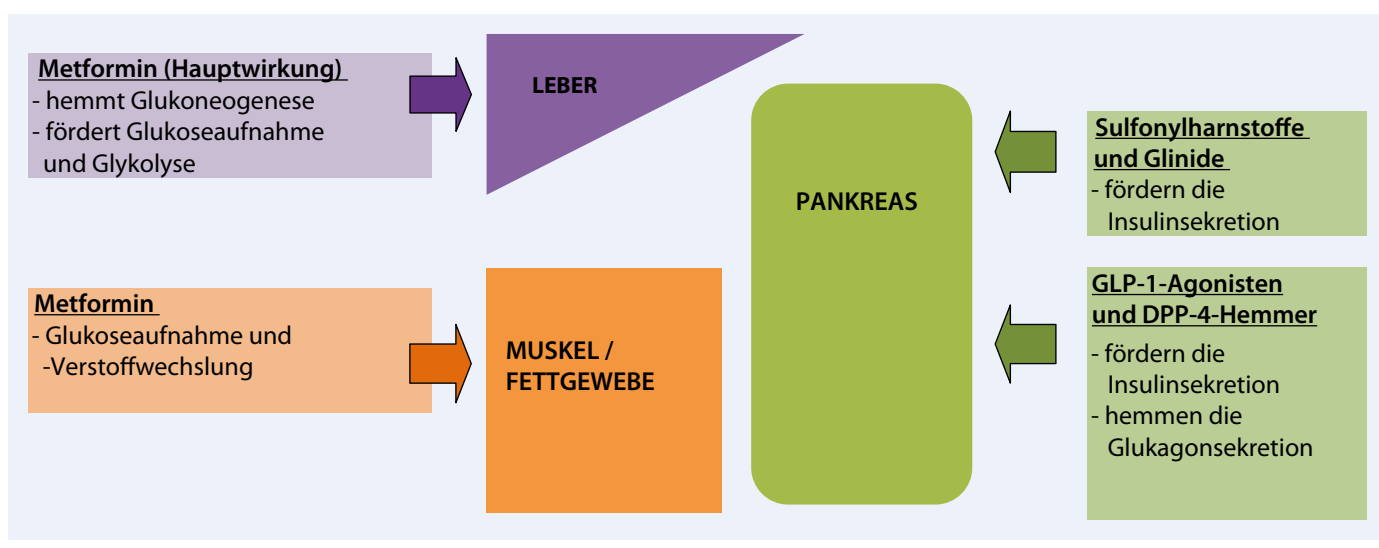

Abb. $1<$ Wirkmechanismen der oralen Antidiabetika

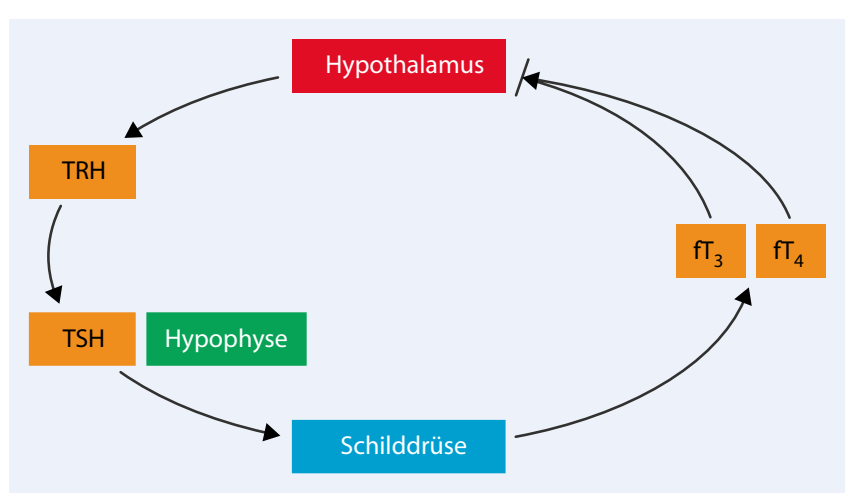

Abb. $2 \Delta$ Im Hypothalamus wird TRH ("thyreotropin releasing hormone") freigesetzt, das die Synthese und Abgabe von TSH (,thyroidea stimulating hormone") aus dem Hypophysenvorderlappen stimuliert. TSH fördert die enterale Jodresorption, die Bildung der Schilddrüsenhormone $\mathrm{T}_{4}$ (Thyroxin) und $\mathrm{T}_{3}$ (Trijodthyronin) und deren Abgabe in die Blutbahn. Sinkt der Blutspiegel der zirkulierenden, freien Schilddrüsenhormone, so wird die Schilddrüse über die Hypothalamus-Hypophysen-Achse zur vermehrten Produktion von Schilddrüsenhormonen angeregt. Bei hohen freien Schilddrüsenhormonen sinkt der zentrale Stimulus über TRH und TSH, und die Hormonproduktion nimmt ab (negativer Feedback). TSH-Rezeptor-stimulierende oder -blockierende Antikörper können Regelkreis-unabhängig die Schilddrüsenhormonproduktion beeinflussen

kontrollierten Studien ein gesicherter klinischer Nutzen einer DHEA-Substitution dokumentiert werden [8]. Es gibt lediglich Hinweise, dass eine DHEA-Substitution bei Frauen mit primärer oder sekundärer Nebenniereninsuffizienz zu einer verbesserten Lebensqualität führen kann [1].

\section{Hypophyse}

Hauptfunktion der Hypophyse ist die Anpassung des Organismus an die bestehende Umgebung und die Reproduktion. Im Wesentlichen reguliert sie Hormone der endokrinen Organe.

Neben der Menopause vermindert sich auch die Wachstumshormonproduktion mit zunehmendem Alter, parallel sinken die Wachstumshormon-abhängigen IGF1-Spiegel (- Tab. 4). Bis heute sind keine klinisch relevanten altersabhängigen Veränderungen der TSH- oder Prolaktinproduktion bekannt (• Tab.4).

\section{Inzidentalome}

Inzidentalome sind Veränderungen der Hypophyse, die als Nebenbefund einer ZNS-Bildgebung erhoben werden. Diese Konstellation nimmt im Alter zu. Wie bei allen hypophysären Veränderungen gilt es, eine autonome Sekretion klinisch und laborchemisch auszuschließen und sicherzustellen, dass keine lokalen Komplikationen (Gesichtsfeldeinschränkung) zu befürchten sind. Eine operative The- rapie - oder alternativ eine Radiotherapie - muss bei dokumentiertem Wachstum und/oder Gefahr von lokalen Komplikationen erwogen werden. Ansonsten können maximal jährliche Verlaufs-MRI durchgeführt werden.

\section{Hypophysenadenome (sezernierende und nicht sezernierende)}

Hypophysenadenome sind gutartige Tumoren des Hypophysenvorderlappens. Die Inzidenz hat in allen Altersgruppen in der letzten Zeit durch die verbesserte Bildgebung (MRI) zugenommen. Heute sind die Hypophysenadenome die dritthäufigste intrazerebrale Neoplasie mit einer Inzidenz von ca. 11/100.000/Jahr [6]. Klinisch manifestieren sich diese Tumoren mit lokalen Komplikationen, d. h. Gesichtsfeldeinschränkungen und/oder Kopfschmerzen und/oder Zeichen der Hypophyseninsuffizienz. Etwa 80\% dieser Adenome sind klinisch nicht sezernierend, bei den übrigen handelt es sich um sezernierende, in erster Linie Wachstumshormon und Prolaktin, selten ACTH sezernierende Adenome.

\section{I) Heute sind die Hypophysenadenome die dritthäufigste intrazerebrale Neoplasie}

Neuere Daten zeigen, dass auch bei älteren Patientinnen mit Hypophysenadenom noch operiert werden soll, falls lokale Komplikationen bestehen. In spezialisierten Zentren ist die Intervention durch die transsphenoidale Route scho- 


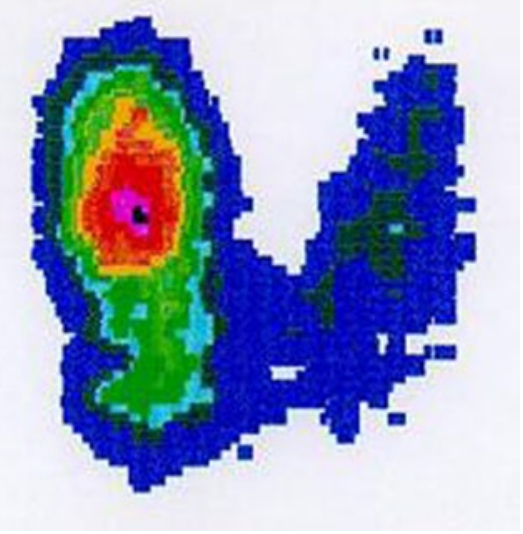

Abb. $3 \Delta$ Autonomie ("toxisches Adenom“). Szintigraphisch stellt sich das toxische Adenom aufgrund der intensiven Jod ${ }^{131}$-Aufnahme als "heißer" Bezirk dar (hier im rechten kranialen Schilddrüsenlappen), während das restliche Schilddrüsengewebe supprimiert ist und daher praktisch keine Radionuklidanreicherung zeigt. Sonographisch imponiert die Autonomie als Knoten. (Abbildung: Autor, mit freundl. Genehmigung)

nend und sicher [7]. Bei sezernierenden Hypophysenadenomen unterscheidet sich der therapeutische Ansatz nicht vom Ansatz bei jüngeren Patientinnen: Prolaktin sezernierende Adenome werden mit Dopamin-Agonisten, Wachstumshormon sezernierende Adenome operativ und bei Bedarf - medikamentös oder als letzte Möglichkeit mit Radiotherapie angegangen. Dopamin-Agonisten sind Gegenspieler von Neuroleptika, die gehäuft auch im Alter eingesetzt werden. Dementsprechend kann es bei einer Therapie mit Dopamin-Agonisten zu einer Verschlimmerung einer psychiatrischen Krankheit kommen. Andererseits verursachen Neuroleptika und gewisse SSRI-Hemmer per se eine Prolaktinerhöhung (ca. 3bis 5-Fache der oberen Norm), was in der Differenzialdiagnose des erhöhten Prolaktins zu berücksichtigen ist. Allgemein gilt, dass nur eine Erhöhung des Prolaktins um das ca. 15- bis 20-Fache der oberen Norm bei bildgebend nachgewiesenem Hypophysenadenom sicher als Prolaktin sezernierendes Adenom zu interpretieren ist.

\section{Hypophysenvorderlappen- insuffizienz}

Eine Hypophysenvorderlappeninsuffizienz (HVL-Insuffizienz) kann Folge von
Hypophysenraumforderungen (und deren Therapie), Traumata oder vaskulärer Genese (Apoplexie) sein. Traumata sind meist mit einer Hypophysenstielpathologie vergesellschaftet, und Apoplexien zeigen eine sehr akute Klinik (Kopfschmerzen und Zeichen des ACTH-Mangels). Die HVL-Insuffizienz kann partiell oder total (Panhypopituitarismus) sein. Die Symptome sind unspezifisch, d. h. Müdigkeit, Leistungsintoleranz, Verminderung der Muskelmasse und Erhöhung der Fettmasse. Diese Symptome sind schwierig von Symptomen des Alterungsprozesses abzugrenzen.

Bei partieller oder kompletter HVLInsuffizienz wird prinzipiell das Hormon der peripheren endokrinen Drüse (d. h. Cortisol, Thyroxin) ersetzt. Bei schwerem Wachstumshormonmangel kann eine Ersatztherapie erwogen werden (Verbesserung der Muskelmasse, Reduktion der Fettmasse), wobei es zu bedenken gilt, dass in epidemiologischen Studien gezeigt wurde, dass der Wachstumshormon-abhängige Marker IGF-1 mit dem Auftreten von verschiedenen Karzinomen korreliert [12].

\section{Hypophysenhinterlappen- insuffizienz}

Klinisch relevant für Patientinnen über 70 Jahre ist die Klinik des Diabetes insipidus, der gerade bei älteren Patientinnen mit vermindertem Durstgefühl zu gefährlichen Hypernatriämien führen kann. Bei isoliert auftretendem Diabetes insipidus muss in diesem Patientengut immer an hämatogene Metastasen im Hypophysenhinterlappen einer anderen Neoplasie (z. B. Mamma-, Lungenkarzinom, Melanom u. a.) gedacht werden. Diese muss dann gezielt gesucht, respektive ausgeschlossen werden.

Therapeutisch wird ein ADH-Analogon eingesetzt, das meist per Nasenspray, selten sublingual oder per subkutaner Injektion abgegeben werden kann.

\section{Fazit für die Praxis}

- Die häufigsten endokrinen Pathologien bei der Frau über 70 Jahre sind das metabolische Syndrom mit/ohne
Typ-2-Diabetes sowie Schilddrüsenstörungen.

- Alle übrigen endokrinen Pathologien sind selten, führen aber wegen langer Latenz der Diagnose häufig zu einer Einschränkung der Lebensqualität.

- Deshalb scheinen uns Kenntnisse der möglichen endokrinen Pathologien von Patientinnen über 70 Jahre wichtig, da sowohl Abklärung als auch Therapie häufig einfach sind und eine lange Leidenszeit beenden.

\section{Korrespondenzadresse}

Prof. Dr. E. Christ

Poliklinik für Endokrinologie, Diabetologie und Klinische Ernährung, Inselspital,

Universitätsspital Bern

3010 Bern

Schweiz

emanuel.christ@insel.ch

\section{Einhaltung ethischer Richtlinien}

Interessenkonflikt. K. Feller-Zulueta und E. Christ geben an, dass kein Interessenkonflikt besteht.

Dieser Beitrag beinhaltet keine Studien an Menschen oder Tieren.

\section{Literatur}

1. Arlt W et al (1999) Dehydroepiandrosterone replacement in women with adrenal insufficiency. $\mathrm{N}$ Engl J Med 341:1013-1020

2. Bauer JH (1995) Age-related changes in the reninaldosterone system. Physiological effects and clinical implications. Drugs Aging 3(3):238

3. Cooper DS (2005) Antithyroid drugs. N Engl J Med 352(9):905

4. Greendale GA et al (1999) The relation between cortisol excretion and fractures in healthy older people: results from the MacArthur studies-Mac. J Am Geriatr Soc 47(7):799

5. Greenspan S (2011) Geriatric endocrinology. In: Gardner D, Shoback D et al (Hrsg) Greenspan's basics \& clinical endocrinology. McGraw-Hill Lange, S 737-762

6. Kuratsu J et al (1997) Epidemiological study of primary intracranial tumours in elderly people. J Neurol Neurosurg Psych 63:116-118

7. Minitti G et al (2005) Diagnosis and management of pituitary tumours in the elderly: a review based on personal experience and evidence of literature. Eur J Endocrinol 153:723-735

8. Nair KS et al (2006) DHEA in elderly women and DHEA or testosterone in elderly men. $\mathrm{N}$ Engl J Med 355(16):1647

9. Nippoldt TB et al (1998) Is there a case for DHEA replacement? Baillieres Clin Endocrinol Metab 12(3):507

10. Noth D et al (2009) Diabetes mellitus und Alter. Ther Umsch 66:707-711 
11. Prague JK et al (2013) Cushing's syndrome. BMJ 346:f945

12. Renehan AG et al (2004) Insulin-like growth factor (IGF)-I, IGF binding protein-3, and cancer risk: systematic review and meta-regression analysis. Lancet 363(9418):1346-1353

13. Schindler AE (2003) Thyroid function and postmenopause. Gynecol Endocrinol 17(1):79

14. Seeman TE et al (1997) Increase in urinary cortisol excretion and memory declines: MacArthur studies of successful aging. J Clin Endocrinol Metab 82(8):2458

15. Surks Ml et al (2004) Subclinical thyroid disease: scientific review and guidelines for diagnosis and management. JAMA 291(2):228

16. Van Cauter E et al (1996) Effects of gender and age on the levels and circadian rhythmicity of plasma cortisol. J Clin Endocrinol Metab 81(7):2468
17. Weetman AP et al (1994) Autoimmune thyroid disease: further developments in our understanding. Endocr Rev 15(6):788

18. World Health Organization, Genf. http://www. who.int/ageing/publications/global_health.pdf

19. Young MJ et al (1993) A multicentre study of the prevalence of diabetic peripheral neuropathy in the United Kingdom hospital clinic population. Diabetologia 36(2):150

20. Zahnd R et al (2013) Aktuelle Diabetes-Therapie. Inf Arzt 3:27-31

\section{Hier steht eine Anzeige.}

\section{Springer}

